07,10

\title{
Аналитические методы расчета упругого взаимодействия точечных дефектов с дислокационными петлями в гексагональных кристаллах
}

\author{
() П.Н. Остапчук, О.Г. Троценко
}

Институт электрофизики и радиационных технологий НАН Украины, Харьков, Украина

E-mail: ostapchuk@kipt.kharkov.ua

(Поступила в Редакцию 26 сентября 2016 г.)

Методом функций Грина для гексагональных кристаллов в подходах Лифшица-Розенцвейга (1947) и Кренера (1953) получены аналитические выражения энергии упругого взаимодействия радиационных точечных дефектов с дислокационными петлями трех типов: базисной краевой ( $c$-петля); базисной сдвиговой и краевой $a$-петлей (плоскость залегания $\{11 \overline{2} 0\}$, вектор Бюргерса $\mathbf{b}^{\mathbf{D}}=1 / 3\langle 11 \overline{2} 0\rangle$ ). В случае базисной краевой петли аналогичное выражение получено независимо решением уравнений равновесия методом Элиота. Численное сравнение полученных выражений для циркония показало полную идентичность рассмотренных подходов.

DOI: $10.21883 /$ FTT.2017.05.44380.361

\section{1. Введение}

Одна из причин эволюции микроструктуры в кристалле под облучением связана с диффузионными потоками радиационных точечных дефектов (ТД) на ее структурные элементы (поры, дислокации, выделения новых фаз и др.), являющиеся стоками для ТД. Сами же потоки существенным образом зависят от градиента энергии упругого взаимодействия (drift flow) ТД с полем напряжений, создаваемым стоком. Дислокационные петли, как правило, - первые наблюдаемые протяженные элементы микроструктуры материала под облучением. Поэтому расчет их упругих полей и соответствующей энергии взаимодействия с ТД представляет собой важнейшую задачу теории радиационного материаловедения.

Если известно фиктивное распределение объемных сил $f_{i}^{S}$, которое создает в упругой среде такие же напряжения, как и действительный источник $S$, то энергию взаимодействия между двумя системами внутренних напряжений $S\left(\mathbf{u}^{S}, u_{i j}^{S}, \sigma_{i j}^{S}\right)$ и $T\left(\mathbf{u}^{T}, u_{i j}^{T}, \sigma_{i j}^{T}\right)$, согласно Эшелби [1], можно представить интегралом вида

$$
E_{\text {int }}(S, T)=-\int f_{i}^{S} u_{i}^{T} d V
$$

который берется по области, содержащей только источник системы $S$. Пусть эта система обусловлена точечным дефектом (ТД), описываемым в теории упругости объемным распределением дипольных сил без момента

$$
f_{i}\left(\mathbf{r}-\mathbf{r}_{0}\right)=-A_{i j} \nabla_{j} \delta\left(\mathbf{r}-\mathbf{r}_{0}\right), \quad A_{i j}=A_{j i},
$$

а система $T-$ дислокационной петлей $(T=D)$. Тогда имеем

$$
E_{\text {int }}(\mathbf{r})=-\int f_{i}\left(\mathbf{r}^{\prime}-\mathbf{r}\right) u_{i}^{D}\left(\mathbf{r}^{\prime}\right) d \mathbf{r}^{\prime}=-A_{i j} u_{i j}^{D}(\mathbf{r}),
$$

а в случае центра дилатации $A_{i j}=P \delta_{i j}$

$$
E_{\text {int }}(\mathbf{r}) / P=-S p u_{i j}^{D}(\mathbf{r}),
$$

где $P$ - мощность центра дилатации; система отсчета при этом связана с дислокацией, a $\mathbf{r}$ - это координата точки нахождения ТД. Собственно методов расчета упругого поля петли $u_{i j}^{D}$ два. Первый - это решение уравнений равновесия в терминах смещений с соответствующими граничными условиями. В случае гексагонального кристалла (осевая симметрия) используется преобразование Ханкеля [2]. Второй - метод тензорной функции Грина $G_{i j}$ (ТФГ) уравнений равновесия данной упругой среды, позволяющей вычислить смещения, создаваемые дислокацией любой формы в любой анизотропной упругой среде, согласно классической формуле $[3,4]$

$$
u_{i}^{D}(\mathbf{r})=C_{j k l m} b_{m}^{D} \int_{S_{D}} n_{l}^{D} \frac{\partial G_{i j}\left(\mathbf{r}-\mathbf{r}^{\prime}\right)}{\partial x_{k}} d S^{\prime} .
$$

Обозначения в (5) следующие: $C_{j k l m}-$ тензор упругих модулей среды, моделирующей кристалл; $b_{m}^{D}-m$-я компонента вектора Бюргерса дислокации; $n_{l}^{D}-l$-я компонента вектора нормали к произвольной поверхности $S_{D}$, опирающейся на дислокационную линию; $\mathbf{r}-$ координата точки наблюдения; $\mathbf{r}^{\prime}-$ координата точки поверхности $S_{D}$. В литературе цитируется два варианта аналитического расчета ТФГ: подход Лифшица-Розенцвейга (1947) [5] и Кренера (1953) [6] с поправкой на коэффициент [7]. Первый справедлив для любой неограниченной упругоанизотропной среды. В частности, применительно к кубическим и гексагональным кристаллам он достаточно подробно изложен в работах [8,9]. Второй применим только к гексагональным кристаллам. 
Чтобы иметь возможность использовать все аналитические методы и сравнивать результаты, в работе рассматриваются кристаллы гексагональной сингонии (численное сравнение проведено для циркония). Точечный дефект моделируется центром дилатации, поэтому для разных петель вычисляется только величина $S p u_{i j}^{D}(\mathbf{r})$, согласно формуле (4). В разделах 2, 3 рассмотрены вакансионные базисные петли с чисто краевой и чисто сдвиговой составляющими вектора Бюргерса. А поскольку наклонный (к базисной плоскости) вектор Бюргерса всегда можно разложить на эти компоненты, то фактически аналитический результат можно получить для любой базисной петли. Несмотря на огромные внешние различия полученных разными методами формул, численные оценки для циркония показали их полную идентичность. В разделе 4 рассмотрен более сложный объект, а именно, петля, залегающая в плоскости $\{11 \overline{2} 0\}$. В частности для циркония, это так называемые $a$-петли с вектором Бюргерса $\mathbf{b}=1 / 3\langle 11 \overline{2} 0\rangle$. Здесь работает только метод ТФГ. И снова численные оценки результатов для циркония, как по Лифшицу-Розенцвейгу, так и по Кренеру, показали их полную идентичность. Следует отметить, что в связи с интерпретацией экспериментально наблюдаемых контрастов от петель, задачи расчета их упругих полей решались неоднократно и ранее (см., например, $[10,11])$. Но, как правило, результат формулировался в виде довольно громоздких формул для компонент напряжений. Нас же интересует только $S p u_{i j}^{D}(\mathbf{r})$, поэтому все приведенные в работе формулы не только оригинальны, но и, как оказалось, намного проще математически.

\section{2. Упругое поле призматической дислокационной петли, лежащей в базисной плоскости гексагонального кристалла}

Рассмотрим круговую вакансионную петлю радиуса $R$, лежащую в плоскости $z=0$ (базисная плоскость) цилиндрической системы координат $(r, \varphi, z)$, вектор Бюргерса которой перпендикулярен плоскости петли и имеет только $z$-компоненту $\left(0,0, b^{D}\right)$. Вектор нормали к плоскости петли совпадает с положительным направлением оси „z“, являющейся также осью симметрии кристалла. Поскольку задача аксиально-симметрична, угловая зависимость отсутствует $u_{\varphi}=0, \sigma_{r \varphi}=\sigma_{\varphi z}=0$, и напряженное состояние однозначно определяется четырьмя компонентами тензора напряжений: $\sigma_{r r}, \sigma_{\varphi \varphi}, \sigma_{z z}$, $\sigma_{r z}$, которые в терминах смещений имеют вид

$$
\begin{gathered}
\sigma_{r r}=C_{11} \frac{\partial u_{r}}{\partial r}+C_{12} \frac{u_{r}}{r}+C_{13} \frac{\partial u_{z}}{\partial z}, \\
\sigma_{\varphi \varphi}=C_{12} \frac{\partial u_{r}}{\partial r}+C_{11} \frac{u_{r}}{r}+C_{13} \frac{\partial u_{z}}{\partial z}, \\
\sigma_{z z}=C_{13}\left(\frac{\partial u_{r}}{\partial r}+\frac{u_{r}}{r}\right)+C_{33} \frac{\partial u_{z}}{\partial z}, \\
\sigma_{r z}=C_{44}\left(\frac{\partial u_{r}}{\partial z}+\frac{\partial u_{z}}{\partial r}\right)
\end{gathered}
$$

и удовлетворяют уравнениями равновесия

$$
\frac{\partial \sigma_{r r}}{\partial r}+\frac{\partial \sigma_{r z}}{\partial z}+\frac{\sigma_{r r}-\sigma_{\varphi \varphi}}{r}=0, \quad \frac{\partial \sigma_{r z}}{\partial r}+\frac{\partial \sigma_{z z}}{\partial z}+\frac{\sigma_{r z}}{r}=0,
$$

где $C_{11}, C_{12}, C_{13}, C_{33}, C_{44}=C_{55}-$ минимальное число отличных от нуля упругих модулей. Примером объекта такого рода могут служить так называемые $c$-петли $\mathbf{b}=1 / 2[0001]$, наблюдавшиеся в цирконии при электронном облучении при $T=715 \mathrm{~K}$. Как показал H.A. Elliot [12], задача расчета упругого поля петли (6), (7) решается в терминах двух функций напряжения $\Phi_{\alpha}(\alpha=1,2)$, которые удовлетворяют уравнениям

$$
\left(\nabla^{2}+v_{\alpha} \frac{\partial^{2}}{\partial z^{2}}\right) \Phi_{\alpha}(r, z)=0, \quad \nabla^{2}=\frac{\partial^{2}}{\partial r^{2}}+\frac{1}{r} \frac{\partial}{\partial r},
$$

а коэффициенты $v_{\alpha}$ являются корнями квадратного уравнения

$C_{44} C_{11} v^{2}+\left(C_{13}^{2}+2 C_{44} C_{13}-C_{33} C_{11}\right) v+C_{44} C_{33}=0$

При этом

$$
\begin{gathered}
u_{r}=\frac{\partial}{\partial r}\left(\Phi_{1}+\Phi_{2}\right), \quad u_{z}=\frac{\partial}{\partial z}\left(k_{1} \Phi_{1}+k_{2} \Phi_{2}\right), \\
k_{\alpha}=\frac{C_{11} v_{\alpha}-C_{44}}{C_{13}+C_{44}}=\frac{v_{\alpha}\left(C_{13}+C_{44}\right)}{C_{33}-C_{44} v_{\alpha}} .
\end{gathered}
$$

Подстановка (10) в (6) дает поле напряжений петли в терминах $\Phi_{\alpha}$, а искомая величина $S p u_{i j}^{D}$ принимает вид

$$
S p u_{i j}^{D}=\sum_{\alpha=1}^{2}\left(k_{\alpha}-v_{\alpha}\right) \frac{\partial^{2}}{\partial z^{2}} \Phi_{\alpha} .
$$

С помощью преобразования Ханкеля $G(\xi, z)=$ $=\int_{0}^{\infty} r \Phi(r, z) J_{0}(\xi r) d r$ [2] уравнения (8) сводятся к обыкновенным дифференциальным уравнениям вида

$$
\left(v_{\alpha} \frac{d^{2}}{d z^{2}}-\xi^{2}\right) G_{\alpha}(\xi, z)=0
$$

решение которых, тривиально $G_{\alpha}(\xi, z)=A_{\alpha}(\xi)$ $\times \exp \left(-\xi z / \sqrt{v_{\alpha}}\right)+B_{\alpha}(\xi) \exp \left(\xi z / \sqrt{v_{\alpha}}\right)$. Поскольку все компоненты смещений и напряжений должны исчезать при $z \rightarrow \infty$, то $B_{\alpha}=0$, а коэффициенты $A_{\alpha}$ определяются из граничных условий задачи. В нашем случае они таковы [13]

$$
\begin{gathered}
u_{z}(r, 0)=-\frac{1}{2} b^{D}, \quad 0 \leq r<R, \\
u_{z}(r, 0)=0, \quad r>R, \quad \sigma_{r z}(r, 0)=0 .
\end{gathered}
$$

Второе условие в (13) следует из чисто краевой природы петли. Знак для $z$-компоненты смещений $u_{z}$ выбран положительным для междоузельной петли и отрицательным для вакансионной. Используя инверсионную теорему Ханкеля [2], и выражение для $G_{\alpha}(\xi, z)$, получаем

$$
\begin{aligned}
\sigma_{r z}(r, z)= & C_{44} \int_{0}^{\infty} \xi^{3} \sum_{\alpha=1}^{2}\left[\frac{\left(1+k_{\alpha}\right)}{\sqrt{v_{\alpha}}} A_{\alpha}(\xi) \exp \left(-\xi z / \sqrt{v_{\alpha}}\right)\right] \\
& \times J_{1}(r \xi) d \xi .
\end{aligned}
$$


И тогда из второго граничного условия имеем связь

$$
A_{2}(\xi)=-A_{1}(\xi) \frac{\left(1+k_{1}\right)}{\left(1+k_{2}\right)} \frac{\sqrt{v_{2}}}{\sqrt{v_{1}}} .
$$

Аналогичным образом для $z$-компоненты смещений $u_{z}$ получаем

$u_{z}(r, z)=-\int_{0}^{\infty} \xi^{2} \sum_{\alpha=1}^{2}\left[\frac{k_{\alpha}}{\sqrt{v_{\alpha}}} A_{\alpha}(\xi) \exp \left(-\xi z / \sqrt{v_{\alpha}}\right)\right] J_{0}(r \xi) d \xi$

и в безразмерных переменных $t=\xi R, \rho=r / R$ согласно первому граничному условию имеем два интегральных уравнения

$$
\int_{0}^{\infty} t^{2} A_{1}(t / R) J_{0}(t \rho) d t=\sqrt{v_{1}} \frac{1+k_{2}}{k_{1}-k_{2}} \frac{b^{D} R^{3}}{2} \quad 0 \leq \rho \leq 1
$$

$$
\int_{0}^{\infty} t^{2} A_{1}(t / R) J_{0}(t \rho) d t=0 \quad \rho>1 .
$$

Они удовлетворяются при

$$
t^{2} A_{1}(t / R)=\sqrt{v_{1}} \frac{1+k_{2}}{k_{1}-k_{2}} \frac{b^{D} R^{3}}{2} J_{1}(t) .
$$

В результате функции напряжения $\Phi_{\alpha}$ принимают вид

$$
\begin{aligned}
\Phi_{1}(r, z)= & \sqrt{v_{1}} \frac{1+k_{2}}{k_{1}-k_{2}} \frac{b^{D} R}{2} \\
& \times \int_{0}^{\infty} \frac{1}{\xi} J_{1}(\xi R) J_{0}(r \xi) \exp \left(-\xi z / \sqrt{v_{1}}\right) d \xi \\
\Phi_{2}(r, z)=- & \sqrt{v_{2}} \frac{1+k_{1}}{k_{1}-k_{2}} \frac{b^{D} R}{2} \\
& \times \int_{0}^{\infty} \frac{1}{\xi} J_{1}(\xi R) J_{0}(r \xi) \exp \left(-\xi z / \sqrt{v_{2}}\right) d \xi .
\end{aligned}
$$

Подставляя (16) в (10) и (6), мы получаем все компоненты упругих напряжений. Выписывать их в явном виде не будем. Это сделано в работе [10]. Нас интересует только $S p u_{i j}^{D}$. Из (11) и (16) имеем

$$
\begin{aligned}
S p u_{i j}^{D}= & \frac{b^{D}}{2 R}\left[\frac{1+k_{2}}{k_{1}-k_{2}} \frac{k_{1}-v_{1}}{\sqrt{v_{1}}} I_{0}^{1}\left(\frac{r}{R}, \frac{z}{R \sqrt{v_{1}}}\right)-\frac{1+k_{1}}{k_{1}-k_{2}}\right. \\
& \left.\times \frac{k_{2}-v_{2}}{\sqrt{v_{2}}} I_{0}^{1}\left(\frac{r}{R}, \frac{z}{R \sqrt{v_{2}}}\right)\right] \equiv \frac{b^{D}}{4 \pi R} I\left(\frac{r}{R}, \frac{z}{R}\right),
\end{aligned}
$$

где

$$
I_{m}^{n}\left(\frac{r}{R}, \frac{z}{R}\right)=\int_{0}^{\infty} t^{n} J_{m}\left(\frac{r}{R} t\right) J_{1}(t) \exp \left(-t \frac{z}{R}\right) d t
$$

И, таким образом, задача расчета энергии упругого взаимодействия центра дилатации с данной призматической петлей решена.
Далее рассмотрим ту же задачу, но в формализме тензорной функции Грина (5)

$$
\begin{aligned}
S p u_{i j}^{D}(\mathbf{r}) & =b^{D} \int_{S_{D}} d^{2} r^{\prime}\left[C_{13} \sum_{\alpha=1}^{2} G_{i \alpha, \alpha i}\left(\mathbf{r}-\mathbf{r}^{\prime}\right)\right. \\
& \left.+C_{33} G_{i 3,3 i}\left(\mathbf{r}-\mathbf{r}^{\prime}\right)\right], \quad G_{i j, k m} \equiv \frac{\partial^{2} G_{i j}}{\partial x_{k} \partial x_{m}} .
\end{aligned}
$$

Применительно к нашей задаче первый метод достаточно подробно изложен в недавней работе авторов [14], поэтому ниже результаты будут приведены лишь конспективно. Метод Кренера был использован в работе Yoо [15] для расчета энергии взаимодействия двух ТД в модели силовых диполей, а его оригинальный способ изложения актуален и в нашем случае. Введем обозначения

$$
K_{1}=\sum_{\gamma=1}^{2} \sum_{\alpha=1}^{2} G_{\gamma \alpha, \alpha \gamma}, \quad K_{2}=G_{33,33}, \quad K_{3}=\sum_{\alpha=1}^{2} G_{3 \alpha, \alpha 3} .
$$

Тогда из (18) следует

$$
S p u_{i j}^{D}=\frac{b^{D}}{4 \pi} \int_{S_{D}} d^{2} r^{\prime}\left[C_{13} K_{1}+C_{33} K_{2}+\left(C_{33}+C_{13}\right) K_{3}\right] .
$$

Согласно Кренеру

$$
\begin{gathered}
G_{11}=\sum_{k=1}^{3} \frac{1}{\left[v_{k} r^{2}+z^{2}\right]^{1 / 2}}\left[A_{k} \frac{x^{2} z^{2}-y^{2}\left(v_{k} r^{2}+z^{2}\right)}{r^{4}}+B_{k}\right], \\
r_{22}=\sum_{k=1}^{3} \frac{1}{\left[v_{k} r^{2}+z^{2}\right]^{1 / 2}}\left[A_{k} \frac{y^{2} z^{2}-x^{2}\left(v_{k} r^{2}+z^{2}\right)}{r^{4}}+B_{k}\right], \\
G_{33}=\sum_{k=1}^{3} \frac{D_{k}}{\left[v_{k} r^{2}+z^{2}\right]^{1 / 2}}, \\
G_{31}=\frac{x z}{r^{2}} \sum_{k=1}^{3} \frac{C_{12}=\frac{x y}{r^{4}} \sum_{k=1}^{3} A_{k} \frac{\left[v_{k} r^{2}+2 z^{2}\right]}{\left[v_{k} r^{2}+z^{2}\right]^{1 / 2}},}{\left[v_{k} r^{2}+z^{2}\right]^{1 / 2}}, \quad G_{32}=\frac{y z}{r^{2}} \sum_{k=1}^{3} \frac{C_{k}}{\left[v_{k} r^{2}+z^{2}\right]^{1 / 2}} .
\end{gathered}
$$

Оказывается, что все функции $K_{i}(i=1,2,3)$ в нашем случае можно записать компактно следующим образом (немного отличающимся от [15])

$$
\begin{gathered}
K_{i}=\frac{1}{\left|\mathbf{r}-\mathbf{r}^{\prime}\right|^{3}} T_{i}\left(\tau_{3}^{2}\right), \quad T_{1}=\sum_{\alpha=1}^{2} A_{\alpha} v_{\alpha}^{2} F_{\alpha}\left(\tau_{3}^{2}\right), \\
T_{2}=\sum_{\alpha=1}^{2} D_{\alpha} F_{\alpha}\left(\tau_{3}^{2}\right), \quad T_{3}=\sum_{\alpha=1}^{2} C_{\alpha} v_{\alpha} F_{\alpha}\left(\tau_{3}^{2}\right), \\
F_{\alpha}\left(\tau_{3}^{2}\right) \equiv \frac{2 \tau_{3}^{2}-v_{\alpha}\left(1-\tau_{3}^{2}\right)}{\left[\tau_{3}^{2}+v_{\alpha}\left(1-\tau_{3}^{2}\right)\right]^{5 / 2}},
\end{gathered}
$$


где $v_{\alpha}$ - корни все того же квадратного уравнения (9), а коэффициенты, фигурирующие в (21) имеют вид

$$
\begin{gathered}
A_{\alpha}=\left[\left(C_{66}-C_{11}\right)\left(C_{33}-v_{\alpha} C_{44}\right)+\left(C_{13}+C_{44}\right)^{2}\right] / E_{\alpha}, \\
C_{66}=\left(C_{11}-C_{12}\right) / 2, \\
D_{\alpha}=\left(C_{44}-v_{\alpha} C_{11}\right)\left(C_{44}-v_{\alpha} C_{66}\right) / E_{\alpha}, \\
C_{\alpha}=\left(C_{13}+C_{44}\right)\left(C_{44}-v_{\alpha} C_{66}\right) / E_{\alpha}, \\
E_{1}=C_{11} C_{44} C_{66}\left(v_{1}-v_{3}\right)\left(v_{1}-v_{2}\right), \\
E_{2}=C_{11} C_{44} C_{66}\left(v_{2}-v_{3}\right)\left(v_{2}-v_{1}\right) .
\end{gathered}
$$

Здесь $\tau_{k}$ - компоненты единичного вектора $\boldsymbol{\tau}=\left(\mathbf{r}-\mathbf{r}^{\prime}\right) /\left|\mathbf{r}-\mathbf{r}^{\prime}\right|$, а также учтено очевидное равенство $\sum_{k} \tau_{k}^{2}=1$. Таким образом, задача сводится к интегрированию (19) с учетом (21), (22) по плоскости круговой петли (напомним, что $z^{\prime}=0$, поскольку петля лежит в базисной плоскости кристалла)

$$
\begin{aligned}
& \operatorname{Spu}_{i j}^{D}(\mathbf{r})=\frac{b^{D}}{4 \pi} \int_{S_{D}} \frac{d^{2} r^{\prime}}{\left|\mathbf{r}-\mathbf{r}^{\prime}\right|^{3}} \\
& \times\left[C_{13} T_{1}\left(\tau_{3}^{2}\right)+C_{33} T_{2}\left(\tau_{3}^{2}\right)+\left(C_{13}+C_{33}\right) T_{3}\left(\tau_{3}^{2}\right)\right] .
\end{aligned}
$$

Такая форма записи (23) выбрана не случайно. Метод Лифшица-Розенцвейга дает аналогичный результат в похожей форме [14] в тех же переменных

$$
\begin{aligned}
S p u_{i j}^{D}(\mathbf{r})= & -\frac{b^{D}}{4 \pi} \int_{S_{D}} \frac{d^{2} r^{\prime}}{\left|\mathbf{r}-\mathbf{r}^{\prime}\right|^{3}} \\
& \times\left[\left(1-3 \tau_{3}^{2}\right) Q\left(\tau_{3}^{2}\right)+2 \tau_{3}^{2}\left(1-\tau_{3}^{2}\right) \frac{d Q}{d \tau_{3}^{2}}\right],
\end{aligned}
$$

$$
Q\left(\tau_{3}^{2}\right)=C_{13} \mathrm{~K}\left(\tau_{3}^{2}\right)+C_{33} W\left(\tau_{3}^{2}\right)+\left(C_{13}+C_{33}\right) V\left(\tau_{3}^{2}\right) .
$$

Функции $\mathrm{K}\left(\tau_{3}^{2}\right), W\left(\tau_{3}^{2}\right)$ и $V\left(\tau_{3}^{2}\right)$ очень громоздкие, поэтому вынесены в Приложение. Важно, что мы имеем три варианта решения одной и той же задачи. Логично их сравнить. Численные оценки были проведены применительно к цирконию. Экспериментальные значения его упругих модулей согласно [16] следующие (Mbar): $C_{11}=1.554, \quad C_{12}=0.672, \quad C_{13}=0.646, \quad C_{33}=1.725$, $C_{55}=C_{44}=0.363$. Результаты сравнения представлены на рис. 1, 2. в безразмерных цилиндрических координатах $\rho=r / R, \xi=z / R$. При этом $\tau_{3}^{2}=\xi^{2} /\left|\mathbf{r}-\mathbf{r}^{\prime}\right|^{2}$, a $\left|\mathbf{r}-\mathbf{r}^{\prime}\right|^{2}=\rho^{2}+\xi^{2}-2 \rho \rho^{\prime} \cos \left(\varphi-\varphi^{\prime}\right)+\rho^{\prime 2}$. Из-за изотропии в базисной плоскости результат естественно не зависит от азимутального угла $\varphi$, поэтому его положили равным нулю. Формулы (17), (23), (24) приведены к единому виду $S p u_{i j}^{D}=\frac{b^{D}}{4 \pi R} I(\rho, \xi)$, поэтому на рисунках $I^{E}(\rho, \xi)$ - соответствует формуле (17) (H.A. Elliot), $I^{K}(\rho, \xi)$ - формуле (23) (Кренер), $I^{L R}(\rho, \xi)-$ формуле (24) (Лифшиц-Розенцвейг). На них показаны зависимости функций $I^{E}, I^{K}, I^{L R}$ от относительного

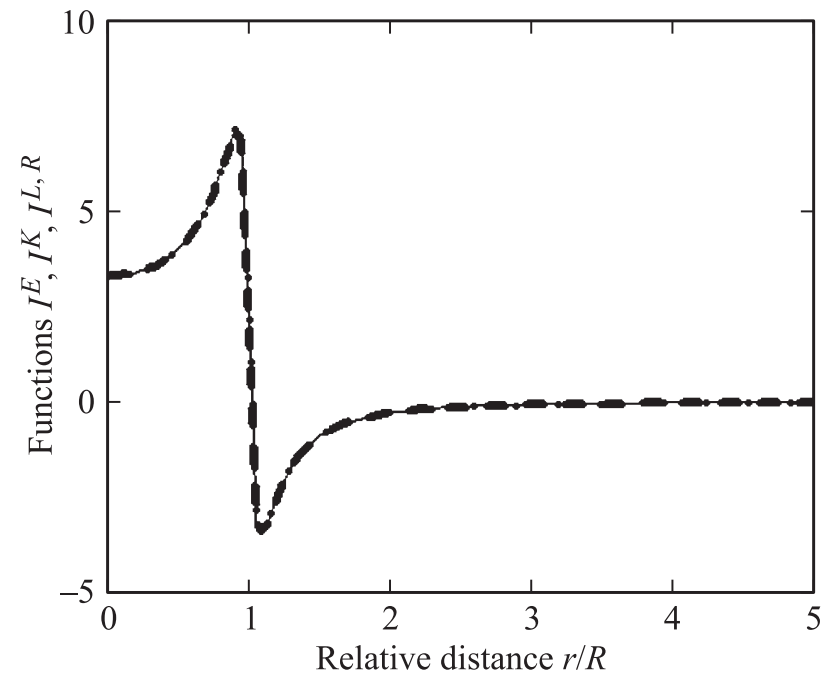

Рис. 1. Функции $I^{E}(\rho, \xi)$ (сплошная линия), $I^{K}(\rho, \xi)$ (пунктирная), $I^{L, R}(\rho, \zeta)$ (штриховая) согласно (17), (23) и (24) в зависимости от относительного расстояния $\rho=r / R$ в плоскости $\xi=0.1$ циркония.

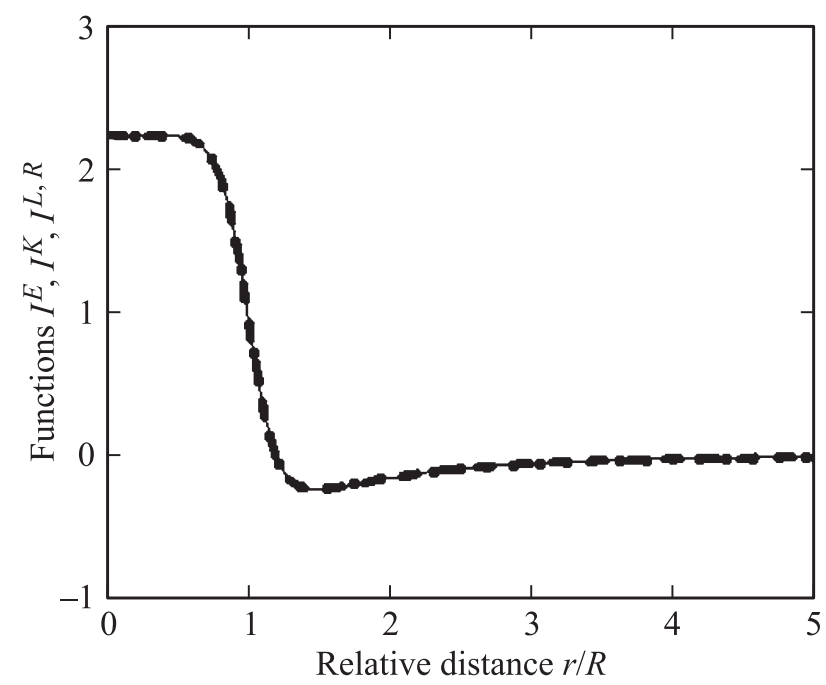

Рис. 2. Функции $I^{E}(\rho, \xi)$ (сплошная линия), $I^{K}(\rho, \xi)$ (пунктирная), $I^{L, R}(\rho, \xi)$ (штриховая) согласно (17), (23) и (24) в зависимости от относительного расстояния $\rho=r / R$ в плоскости $\xi=0.5$ циркония.

расстояния $\rho$ для двух плоскостей $z=0.1 R$ и $z=0.5 R$. Идеальное согласие всех трех вариантов. В предыдущей работе авторов [14] $I^{L R}(\rho, \xi)$ сравнивалась с изотропным приближением. И там различие хоть и небольшое, но было. Здесь его нет. Но это только частный случай. Большинство петель имеют вектор Бюргерса, наклоненный к плоскости петли. А поскольку его всегда можно разложить на призматическую (перпендикулярную плоскости петли) и сдвиговую (лежащую в плоскости петли) компоненты, целесообразно было рассмотреть аналогичную задачу для его чисто сдвиговой составляющей. 


\section{3. Упругое поле сдвиговой дислокационной петли, лежащей в базисной плоскости гексагонального кристалла}

Поскольку аксиальная симметрия задачи в данном случае нарушается, метод преобразований Ханкеля не применим. Остается метод функций Грина. Выберем ось , $x$ “ базисной плоскости , $x y^{“}$ вдоль сдвиговой составляющей $b^{S}$ вектора Бюргерса петли. Тогда из (5) для компонент вектора смещений имеем

$$
u_{i}^{S}(\mathbf{r})=b^{S} C_{44} \int_{S_{D}}\left[G_{i 3,1}\left(\mathbf{r}-\mathbf{r}^{\prime}\right)+G_{i 1,3}\left(\mathbf{r}-\mathbf{r}^{\prime}\right)\right] d^{2} r^{\prime},
$$

где интегрирование, как и ранее, проводится по площади петли. И нас по-прежнему будет интересовать только величина $S p u_{i j}^{S}$

$$
S p u_{i j}^{S}(\mathbf{r})=b^{S} C_{44} \int_{S_{D}}\left[G_{i 3, i 1}\left(\mathbf{r}-\mathbf{r}^{\prime}\right)+G_{i 1, i 3}\left(\mathbf{r}-\mathbf{r}^{\prime}\right)\right] d^{2} r^{\prime}
$$

по индексу „i“ су суммирование от 1 до 3 . Проделав необходимые вычисления, по Кренеру получаем

$$
\begin{aligned}
S p u_{i j}^{S}(\mathbf{r}) & =\frac{b^{S}}{4 \pi} C_{44} \int_{S_{D}} d^{2} r^{\prime} \frac{\tau_{3} \tau_{1}}{\left|\mathbf{r}-\mathbf{r}^{\prime}\right|^{3}} \\
\times & {\left[3 \sum_{\alpha=1}^{2} \frac{\left(C_{\alpha}-A_{\alpha}\right) v_{\alpha}^{2}+\left(D_{\alpha}-C_{\alpha}+B_{\alpha}\right) v_{\alpha}}{\left[v_{\alpha}\left(1-\tau_{3}^{2}\right)+\tau_{3}^{2}\right]^{5 / 2}}\right], }
\end{aligned}
$$

где

$B_{\alpha}=\left[\left(C_{44}-v_{\alpha} C_{11}\right)\left(C_{33}-v_{\alpha} C_{44}\right)+v_{\alpha}\left(C_{13}+C_{44}\right)^{2}\right] / E_{\alpha}$,

а все остальные величины определены выше (22) $\left(\tau_{k}=\left(x_{k}-x_{k}^{\prime}\right) /\left|\mathbf{r}-\mathbf{r}^{\prime}\right|\right)$. Похожая задача решалась в работе [11] в связи с интерпретацией экспериментально наблюдаемых контрастов петель. Результаты в ней приведены для компонент упругих напряжений. Они очень громоздкие и представлены в терминах интегралов $I_{m}^{n}(17)$. Наша формула иная. Она достаточно компактная, легко воспринимаема и, поэтому, мы считаем ее абсолютно оригинальной.

Аналогичное выражение в тех же переменных по Лифшицу-Розенцвейгу имеет вид

$$
\begin{aligned}
& S p u_{i j}^{S}(\mathbf{r})=\frac{b^{S}}{4 \pi} C_{44} \int_{S_{D}} d^{2} r^{\prime} \frac{\tau_{3} \tau_{1}}{\left|\mathbf{r}-\mathbf{r}^{\prime}\right|^{3}} \\
& \quad \times\left[3\left\{\Psi\left(\tau_{3}^{2}\right)-Y\left(\tau_{3}^{2}\right)\right\}+2 \tau_{3}^{2} \frac{d \Psi}{d \tau_{3}^{2}}+2\left(1-\tau_{3}^{2}\right) \frac{d Y}{d \tau_{3}^{2}}\right],
\end{aligned}
$$

$$
\Psi\left(\tau_{3}^{2}\right)=V\left(\tau_{3}^{2}\right)+W\left(\tau_{3}^{2}\right), \quad Y\left(\tau_{3}^{2}\right)=\mathrm{K}\left(\tau_{3}^{2}\right)+V\left(\tau_{3}^{2}\right) .
$$

Функции $\mathrm{K}\left(\tau_{3}^{2}\right), W\left(\tau_{3}^{2}\right)$ и $V\left(\tau_{3}^{2}\right)$ даны в Приложении. В безразмерных цилиндрических координатах $\rho=r / R$;

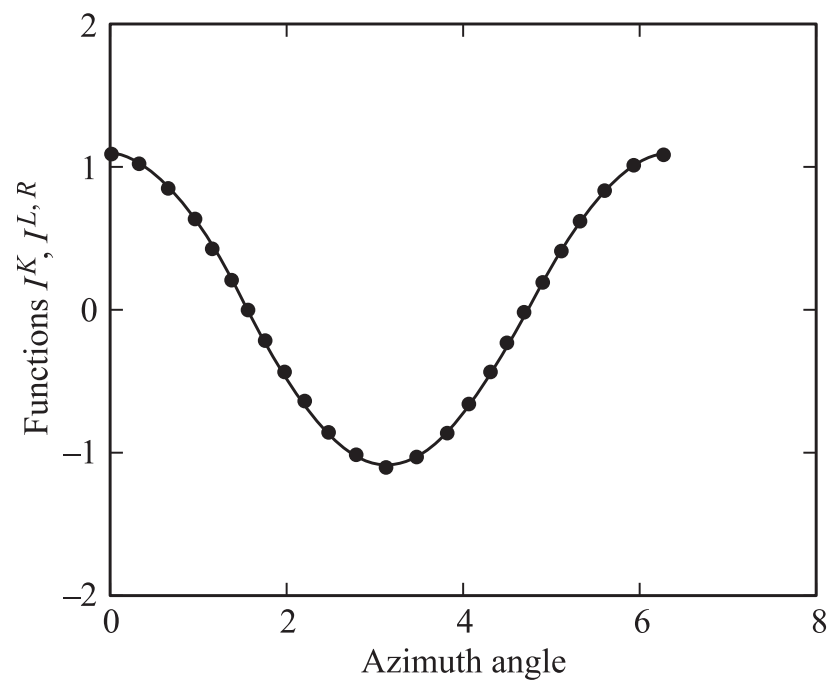

Рис. 3. Зависимость функций $I^{K}(\rho, \xi, \varphi)$ (пунктирная) и $I^{L, R}(\rho, \xi, \varphi)$ (сплошная линия), согласно (27), (28) от азимутального угла $\varphi$ в плоскости $\xi=0.5$ циркония для относительного расстояния $\rho=1.5$.

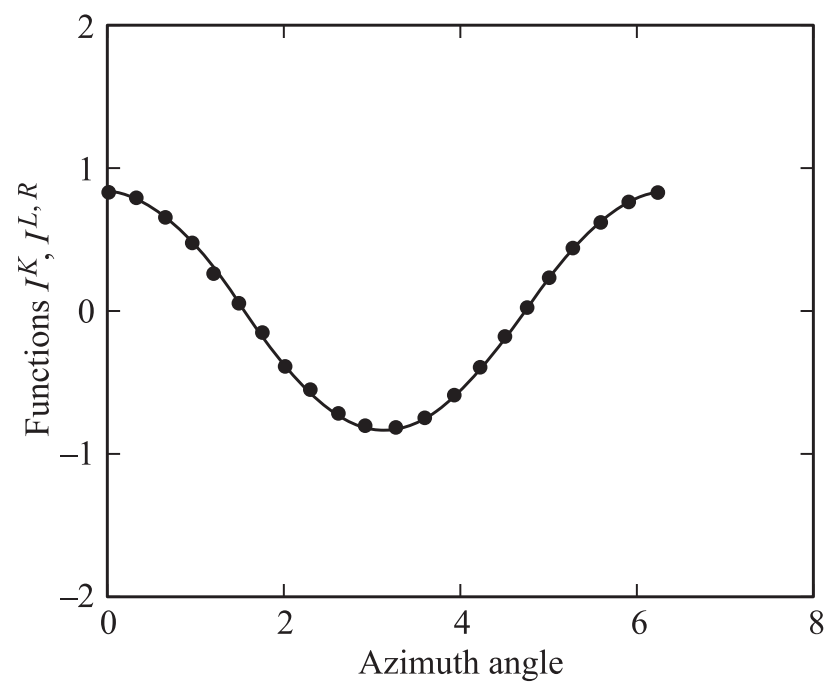

Рис. 4. Зависимость функций $I^{K}(\rho, \xi, \varphi)$ (пунктирная) и $I^{L, R}(\rho, \xi, \varphi)$ (сплошная линия) согласно (27), (28) от азимутального угла $\varphi$ в плоскости $\xi=0.5$ циркония для относительного расстояния $\rho=0.5$.

$\xi=z / R$ для круговой петли формулы (27), (28) снова приводим к виду $S p u_{i j}^{S}=\frac{b^{S}}{4 \pi R} I(\rho, \xi, \varphi)$ и сравниваем численно соответствующие выражения $I^{K}$ по Кренеpy (27) и $I^{L, R}$ по Лифшицу-Розенцвейгу (28). Результаты приведены на рис. 3-5. Во-первых, следует отметить полное совпадение в обоих подходах, что говорит об их эквивалентности. Но в отличие от чисто призматической петли здесь появляется зависимость от азимутального угла $\varphi$ в базисной плоскости гексагонального кристалла. На рис. 3,4 показана такая зависимость функций $I^{K}$ и $I^{L, R}$ в плоскости $z=0.5 R$ циркония для двух значений $r$ : 


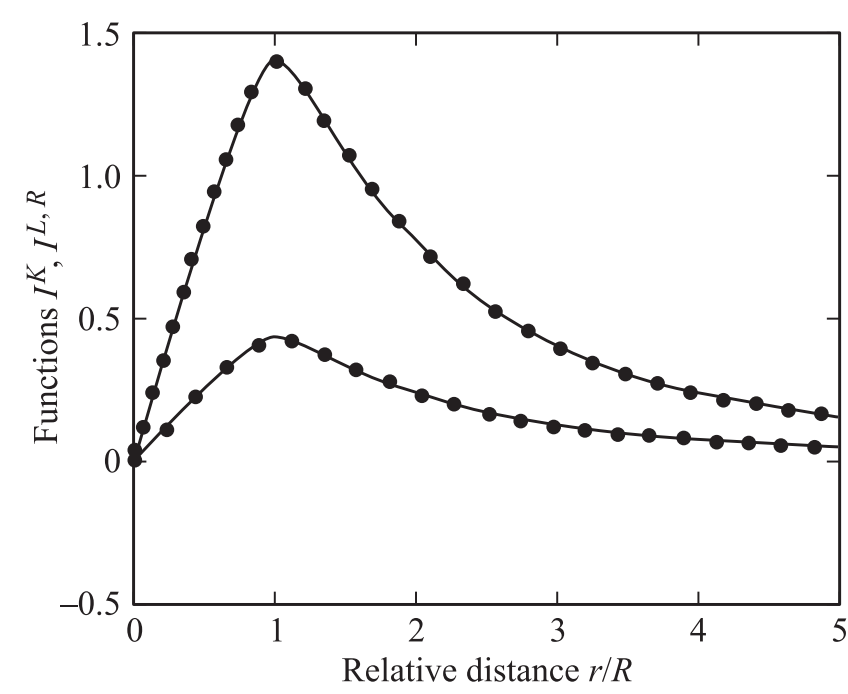

Рис. 5. Зависимость функций $I^{K}(\rho, \xi, \varphi)$ (пунктирная) и $I^{L, R}(\rho, \xi, \varphi)$ (сплошная линия) в плоскости циркония $\xi=0.5$ согласно (27), (28) для двух углов $\varphi=0$ и $\varphi=2 \pi / 5$ от относительного расстояния $\rho=r / R$.

$r=1.5 R$ (вне петли) и $r=0.5 R$ (внутри петли). Видно, что в обоих случаях взаимодействие меняет знак при $\varphi=\pi / 2$ и $\varphi=3 \pi / 2$. Центр дилатации притягивается в одной половине сдвиговой петли и отталкивается в другой. На рис. 5 показана зависимость функций $I^{K}$ и $I^{L, R}$ в плоскости циркония $z=0.5 R$ для двух углов $\varphi=0$ и $\varphi=2 \pi / 5$ от относительного расстояния $\rho=r / R$. Знак взаимодействия один и тот же, поскольку $0 \leq \varphi \leq \pi / 2$, а по абсолютной величине оно уменьшается до нуля при $\varphi=\pi / 2$. Таким образом, вопрос об упругом взаимодействии базисной дислокационной петли гексагонального кристалла с центром дилатации можно считать закрытым.

\section{4. Упругое поле $a$-петли в цирконии}

Следующим объектом нашего исследования являются $a$-петли в цирконии $\mathbf{b}=1 / 3\langle 11 \overline{2} 0\rangle$, залегающие в плоскости $\{11 \overline{2} 0\}$. Это доминирующая форма петель при нейтронном облучении. Это совершенные призматические петли, как вакансионные, так и междоузельные по своей природе [17]. Для вакансионной $a$-петли направления вектора Бюргерса и нормали к плоскости петли совпадают, поэтому ось „х“ декартовой системы координат естественно выбрать в этом же направлении. Тогда смещения из (5) удобно записать в виде

$$
\begin{aligned}
u_{i}^{D}(\mathbf{r})= & b^{D} \int_{S_{D}}\left[C_{12} G_{i \alpha, \alpha}+C_{13} G_{i 3,3}\right] d^{2} r^{\prime} \\
& +b^{D}\left(C_{11}-C_{12}\right) \int_{S_{D}} G_{i 1,1}\left(\mathbf{r}-\mathbf{r}^{\prime}\right) d^{2} r^{\prime} .
\end{aligned}
$$

Соответственно

$$
\begin{aligned}
\operatorname{Spu}_{i j}^{D}(\mathbf{r})= & b^{D} \int_{S_{D}}\left[C_{12} G_{i \alpha, \alpha i}+C_{13} G_{i 3,3 i}\right] d^{2} r^{\prime} \\
& +b^{D}\left(C_{11}-C_{12}\right) \int_{S_{D}} G_{i 1,1 i}\left(\mathbf{r}-\mathbf{r}^{\prime}\right) d^{2} r^{\prime}
\end{aligned}
$$

по индексу „,“ в правой части (30) подразумевается суммирование от 1 до 2 , а по индексу , ,“ от 1 до 3. Удобство такой записи заключается в том, что первое слагаемое в (30) с точностью до коэффициентов совпадает с выражением (18), для которого результат уже известен как по Кренеру (23), так и по Лифшицу-Розенцвейгу (24). Поэтому вычислению подлежит только сумма $G_{i 1,1 i}$. Результат вычислений по Кренеру

$$
\begin{gathered}
S p u_{i j}^{D}(\mathbf{r})=\frac{b^{D}}{4 \pi} \int_{S_{D}} \frac{d^{2} r^{\prime}}{\left|\mathbf{r}-\mathbf{r}^{\prime}\right|^{3}} \\
\times\left[C_{12} T_{1}\left(\tau_{3}^{2}\right)+C_{13} T_{2}\left(\tau_{3}^{2}\right)+\left(C_{12}+C_{13}\right) T_{3}\left(\tau_{3}^{2}\right)\right] \\
+\frac{b^{D}}{4 \pi}\left(C_{11}-C_{12}\right) \int_{S_{D}} \frac{d^{2} r^{\prime}}{\left|\mathbf{r}-\mathbf{r}^{\prime}\right|^{3}} T\left(\tau_{1}^{2}, \tau_{3}^{2}\right) \\
T\left(\tau_{1}^{2}, \tau_{3}^{2}\right)=\sum_{\alpha=1}^{2} \frac{\left[A_{\alpha} v_{\alpha}^{2}+\left(C_{\alpha}-B_{\alpha}\right) v_{\alpha}\right]}{\left[v_{\alpha}\left(1-\tau_{3}^{2}\right)+\tau_{3}^{2}\right]^{3 / 2}} \\
\times\left[1-\frac{3 \tau_{1}^{2} v_{\alpha}}{v_{\alpha}\left(1-\tau_{3}^{2}\right)+\tau_{3}^{2}}\right]
\end{gathered}
$$

где все функции и константы определены выше (21), (22). Аналогичный результат по ЛифшицуРозенцвейгу

$$
\begin{gathered}
S_{p u} u_{i j}(\mathbf{r})=-\frac{b^{D}}{4 \pi} \int_{S_{D}} \frac{d^{2} r^{\prime}}{\left|\mathbf{r}-\mathbf{r}^{\prime}\right|^{3}} Q\left(\tau_{3}^{2}\right) \\
-\frac{b^{D}}{4 \pi}\left(C_{11}-C_{12}\right) \int_{S_{D}} \frac{d^{2} r^{\prime}}{\left|\mathbf{r}-\mathbf{r}^{\prime}\right|^{3}} \tau_{1}^{2}\left[3 Y\left(\tau_{3}^{2}\right)+2 \tau_{3}^{2} \frac{d Y}{d \tau_{3}^{2}}\right], \\
Q\left(\tau_{3}^{2}\right)=\left(1-3 \tau_{3}^{2}\right)\left[C_{12} Y\left(\tau_{3}^{2}\right)+C_{13} \Psi\left(\tau_{3}^{2}\right)\right]+2 \tau_{3}^{2}\left(1-\tau_{3}^{2}\right) \\
\times \frac{d}{d \tau_{3}^{2}}\left[C_{12} Y\left(\tau_{3}^{2}\right)+C_{13} \Psi\left(\tau_{3}^{2}\right)\right]-\left(C_{11}-C_{12}\right) Y\left(\tau_{3}^{2}\right), \\
\Psi\left(\tau_{3}^{2}\right)=V\left(\tau_{3}^{2}\right)+W\left(\tau_{3}^{2}\right), \quad Y\left(\tau_{3}^{2}\right)=\mathrm{K}\left(\tau_{3}^{2}\right)+V\left(\tau_{3}^{2}\right) .
\end{gathered}
$$

Функции $\mathrm{K}\left(\tau_{3}^{2}\right), W\left(\tau_{3}^{2}\right)$ и $V\left(\tau_{3}^{2}\right)$ даны в Приложении. Здесь надо помнить, что петля залегает в плоскости „уз“, т.е. $\tau_{1}=x /\left|\mathbf{r}-\mathbf{r}^{\prime}\right|$, а $\tau_{3}=\left(z-z^{\prime}\right) /\left|\mathbf{r}-\mathbf{r}^{\prime}\right|$. Поэтому безразмерные цилиндрические координаты, используемые для визуального сравнения формул (31) и (32) для круговой петли, следующие: $\xi=x / R, \rho=r / R$ 


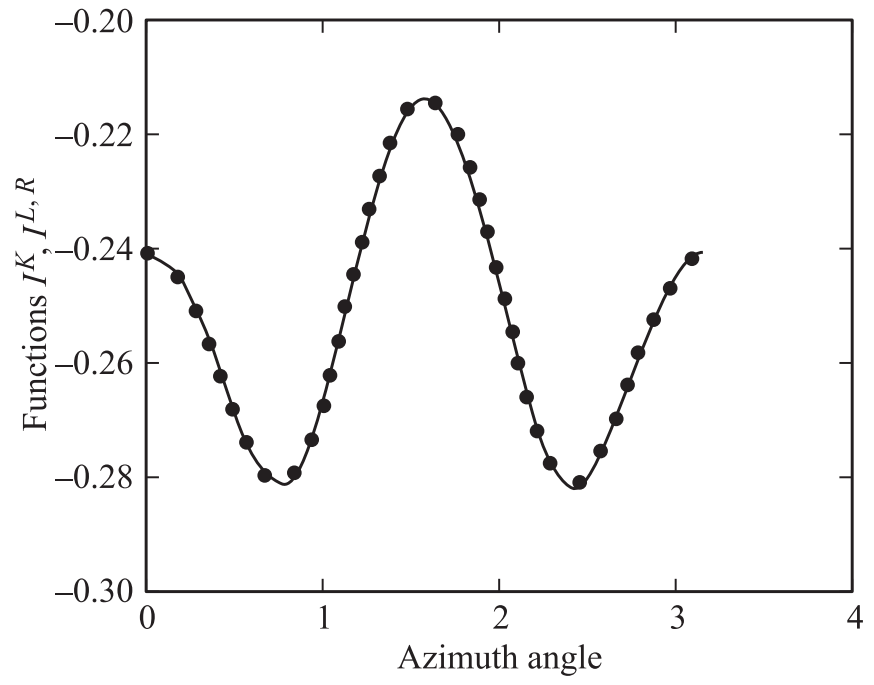

Рис. 6. Зависимость функций $I^{K}(\rho, \xi, \varphi)$ (сплошная линия) и $I^{L, R}(\rho, \xi, \varphi)$ (пунктирная) для $a$-петли циркония согласно (31), (32) от азимутального угла $\varphi$ в плоскости $\xi=0.5$ для относительного расстояния $\rho=1.5$.

$\left(r^{2}=y^{2}+z^{2} ; \quad y=r \cos \varphi ; z=r \sin \varphi\right.$, т. е. $\varphi-$ это по-прежнему азимутальный угол, но в плоскости петли „yz“). Как и ранее, (31), (32) приводим к стандартному виду $S p u_{i j}^{D}=\frac{b^{D}}{4 \pi R} I(\rho, \xi, \varphi)$ и сравниваем численно соответствующие выражения $I^{K}$ по Кренеру (31) и по Лифшицу-Розенцвейгу (32). Для циркония результат приведен на рис. 6 для $\xi=0.5$ и $\rho=1.5$ (область вне петли). И снова полное совпадение в обоих подходах. В отличие от базисной призматической петли у функций $I^{K}$ и $I^{L, R}$ появляется слабая зависимость от азимутального угла $\varphi$, но характер взаимодействия (их знак) от $\varphi$ не зависит. Для $\rho=0.5$ (область над петлей) взаимодействие, как и в случае базисной петли, меняет знак, а слабая угловая зависимость функций $I^{K}, I^{L, R}$ остается. Мы не стали ее приводить, чтобы избежать нагромождения рисунков. Оказывается, что зависимость $I^{K}, I^{L, R}$ от $\rho$ для разных $\xi$ практически совпадает с аналогичной для базисной призматической петли (см. рис.1, 2). И это вполне естественно в силу слабой чувствительности этих функций к углу $\varphi$.

\section{5. Результаты}

Итак, данный материал заключает небольшую серию работ $[8,9,14]$, связанных с методикой вычисления и конкретным применением тензорной функции Грина в подходе Лифшица-Розенцвейга [5]. Для кубических кристаллов этот подход подразумевает разложение по малому параметру анизотропии и согласно $[8,18]$ поправки к [5] весьма существенны. Для гексагональных кристаллов имеет место два метода вычисления ТФГ: Лифшица-Розенцвейга [5,9] и Кренера [6]. В обоих методах компоненты ТФГ имеют универсальный вид, справедливый для любого гексагонального кристалла, однако внешне они настолько различны, что сравнить их можно только по результатам конкретных приложений. В литературе применительно к упругому взаимодействию между двумя ТД или ТД и инфинитезимальной петлей обычно цитируется метод Кренера. Поэтому основная цель работы состояла в том, чтобы сравнить результаты обоих методов на конкретных примерах упругого взаимодействия ТД с дислокационными петлями конечных размеров в реальных ГПУ - металлах.

Рассмотрены три варианта вакансионных петель: базисная призматическая $\mathbf{n}=(0,0,1), \mathbf{b}^{\mathbf{D}}=\left(0,0, b^{D}\right)$; базисная сдвиговая $\mathbf{n}=(0,0,1), \quad \mathbf{b}^{\mathbf{D}}=\left(b^{S}, 0,0\right) \quad$ и призматическая $a$-петля циркония $\mathbf{n}=(1,0,0)$, $\mathbf{b}^{\mathbf{D}}=\left(b^{D}, 0,0\right) \quad$ (плоскость залегания $\{11 \overline{2} 0\}, \quad$ вектор Бюргерса $\left.\mathbf{b}^{\mathbf{D}}=1 / 3\langle 11 \overline{2} 0\rangle\right)$. Для каждого варианта получены аналитические выражения величины $S p u_{i j}^{D}(\mathbf{r})$ (ТД моделируется центром дилатации) обоими методами (23), (24), (27), (28), (31), (32). В случае базисной призматической петли аналогичное выражение получено независимо решением уравнений равновесия методом Эллиота [12] (17). Для сравнения результатов использовалась круговая форма петли. В безразмерных цилиндрических координатах они представимы в виде $S p u_{i j}^{D}=\frac{b^{D}}{4 \pi R} I(\rho, \xi, \varphi)$. Численные расчеты кривых $I(\rho, \xi, \varphi)$ проводились для циркония и представлены на рис. 1-6. Главный вывод - полная эквивалентность всех методов. Однако, учитывая ограниченную применимость [12], реальным расчетным методом остается метод ТФГ в подходах Лифшица-Розенцвейга и Кренера.

Упрощающим обстоятельством в выше рассмотренных случаях дислокационных петель есть то, что вектор нормали к плоскости петли и ее вектор Бюргерса имеют только одну компоненту в декартовой системе координат. В общем случае это не так. И исходные соотношения (5) тогда существенно усложняются. Однако методика расчетов остается прежней и не содержит принципиальных математических трудностей.

Знание энергии упругого взаимодействия ТД с петлей позволяет рассчитать диффузионные потоки радиационных ТД на нее $[19,20]$, а также ее фактор предпочтения (bias) к определенному типу ТД. Последний является важнейшей характеристикой дислокаций, в терминах которой нашли свое объяснение такие явления, как распухание, радиационная ползучесть, радиационное упрочнение конструкционных материалов ядерных реакторов [21-24] и др. Здесь, однако, надо заметить следующее. Во всех теориях фактор предпочтения дислокации рассчитывался в предположении упруго изотропного кристалла, когда энергия взаимодействия ТД с прямолинейной дислокацией или петлей - гармоническая функция. Для гексагонального кристалла это не так. Это следует, например, из выражения (17) для базисной призматической петли. Поэтому соответствующая диффузионная задача $[19,20]$ усложняется наличием допол- 
нительного слагаемого, а выводы авторов относительно зависимости фактора предпочтения от радиуса петли, ее типа и отношения дилатационных объемов ТД, могут поменяться. Но это уже другая задача.

\section{Приложение}

$$
\begin{gathered}
V\left(\tau_{3}^{2}\right) \equiv\left(1-3 \tau_{3}^{2}\right) \Phi\left(\tau_{3}^{2}\right)+2 \tau_{3}^{2}\left(1-\tau_{3}^{2}\right) \frac{d \Phi}{d \tau_{3}^{2}} \\
W\left(\tau_{3}^{2}\right) \equiv F\left(\tau_{3}^{2}\right)-2\left(1-\tau_{3}^{2}\right) \frac{d F}{d \tau_{3}^{2}} \\
\mathrm{~K}\left(\tau_{3}^{2}\right) \equiv-N\left(\tau_{3}^{2}\right)-2 \tau_{3}^{2} \frac{d N}{d \tau_{3}^{2}} \\
-3 \tau_{3}^{2} M\left(\tau_{3}^{2}\right)+2 \tau_{3}^{2}\left(1-\tau_{3}^{2}\right) \frac{d M}{d \tau_{3}^{2}}
\end{gathered}
$$

где функции $\Phi\left(\tau_{3}^{2}\right), F\left(\tau_{3}^{2}\right), N\left(\tau_{3}^{2}\right), M\left(\tau_{3}^{2}\right)$ связаны с компонентами ТФГ соотношениями

$$
\begin{aligned}
& G_{3 k}\left(\mathbf{r}-\mathbf{r}^{\prime}\right)=\frac{1}{4 \pi\left|\mathbf{r}-\mathbf{r}^{\prime}\right|}\left[\Phi\left(\tau_{3}^{2}\right) \tau_{3} \tau_{\beta} \delta_{\beta k}+F\left(\tau_{3}^{2}\right) \delta_{3 k}\right] \\
& \Phi\left(\tau_{3}^{2}\right) \equiv \frac{2 i}{\left(z_{1}+z_{2}\right)} \frac{a+b+\chi+\rho}{A\left(\tau_{3}^{2}\right)}, \\
& F\left(\tau_{3}^{2}\right) \equiv \frac{2 i}{\left(z_{1}+z_{2}\right) A\left(\tau_{3}^{2}\right)} \\
& \times\left((b+\rho)+(a+b-\rho) \tau_{3}^{2}-\frac{a+2 b}{z_{1} z_{2}}\right), \\
& G_{\alpha k}\left(\mathbf{r}-\mathbf{r}^{\prime}\right)=\frac{1}{4 \pi\left|\mathbf{r}-\mathbf{r}^{\prime}\right|} \\
& \times\left[N\left(\tau_{3}^{2}\right) \delta_{\alpha k}-M\left(\tau_{3}^{2}\right) \tau_{\alpha} \tau_{\beta} \delta_{\beta k}+\Phi\left(\tau_{3}^{2}\right) \tau_{\alpha} \tau_{3} \delta_{3 k}\right], \\
& N\left(\tau_{3}^{2}\right) \equiv \frac{2 i}{\left(z_{1}+z_{2}\right)} \frac{R\left(\tau_{3}^{2}\right)}{b A\left(\tau_{3}^{2}\right)}-\frac{b \tau_{3}^{2}}{\sqrt{b P\left(\tau_{3}^{2}\right)}(b+\rho)\left(1-\tau_{3}^{2}\right)}, \\
& M\left(\tau_{3}^{2}\right) \equiv \frac{2 i}{\left(z_{1}+z_{2}\right)} \frac{S\left(\tau_{3}^{2}\right)}{\left(1-\tau_{3}^{2}\right) b A\left(\tau_{3}^{2}\right)} \\
& -\frac{P\left(\tau_{3}^{2}\right)+b \tau_{3}^{2}}{\sqrt{b P\left(\tau_{3}^{2}\right)}(b+\rho)\left(1-\tau_{3}^{2}\right)^{2}} \\
& R\left(\tau_{3}^{2}\right)=\frac{(a+b)(b+\rho)}{z_{1} z_{2}} \\
& -\frac{A\left(\tau_{3}^{2}\right) P\left(\tau_{3}^{2}\right)}{2(b+\rho)\left(1-\tau_{3}^{2}\right)}\left(z_{1} z_{2}-\frac{b}{P\left(\tau_{3}^{2}\right)}\right), \\
& S\left(\tau_{3}^{2}\right)=(a+b)(b+\rho)\left[\frac{1}{z_{1} z_{2}}+\tau_{3}^{2}\right]-\frac{A\left(\tau_{3}^{2}\right) P\left(\tau_{3}^{2}\right)}{2(b+\rho)\left(1-\tau_{3}^{2}\right)} \\
& \times\left[\left(z_{1} z_{2}-\frac{b}{P}\right)+\left(z_{1}^{2} z_{2}^{2}+\frac{b}{P}\left[z_{1} z_{2}-\frac{2 B}{A}\right]\right) \tau_{3}^{2}\right],
\end{aligned}
$$

$$
\begin{gathered}
A\left(\tau_{3}^{2}\right)=2\left[k+l\left(1-\tau_{3}^{2}\right)-m\left(1-\tau_{3}^{2}\right)^{2}\right], \\
B\left(\tau_{3}^{2}\right)=2 k+l\left(1-\tau_{3}^{2}\right), \quad P\left(\tau_{3}^{2}\right)=b+\rho\left(1-\tau_{3}^{2}\right) \\
k=(a+2 b)(b+\rho), \quad m=(a+b-\rho) \gamma-(\chi+2 \rho)^{2}, \\
l=(a+2 b) \gamma+(2 b-\chi)(\chi+2 \rho), \\
a=C_{12}, \quad b=\frac{1}{2}\left(C_{11}-C_{12}\right)=C_{66}, \quad \chi=C_{13}-C_{12}, \\
\rho=C_{44}-\frac{1}{2}\left(C_{11}-C_{12}\right), \quad \gamma=C_{11}+C_{33}-4 C_{44}-2 C_{13}, \\
z_{1} z_{2}=-\sqrt{\frac{2 k}{A\left(\tau_{3}^{2}\right)}}, \quad z_{1}+z_{2}=i \sqrt{2}\left(\sqrt{\frac{2 k}{A\left(\tau_{3}^{2}\right)}}+\frac{B\left(\tau_{3}^{2}\right)}{A\left(\tau_{3}^{2}\right)}\right)^{1 / 2} .
\end{gathered}
$$

\section{Список литературы}

[1] Дж. Эшелби. Континуальная теория дислокаций. Наука, M. (1963). 215 c.

[2] Ian.N. Sneddon. Fourier Transforms. Mc Graw Hill. N.Y. (1951). 542 p.

[3] Л.Д. Ландау, Е.М. Лифшиц. Теория упругости. Наука, М. (1987). $246 \mathrm{c}$.

[4] Дж. Хирт, И. Лоте. Теория дислокаций. Атомиздат, М. (1972). $600 \mathrm{c}$.

[5] И.М. Лифшиц, Л.Н. Розенцвейг. ЖЭТФ 17, 783 (1947).

[6] E. Kröner. Z. Phys. 136, 402 (1953).

[7] J.R. Willis. Quart. J. Mech. Appl. Math. 18, 419 (1965).

[8] П.Н. Остапчук. ФТТ 54, 92 (2012).

[9] П.Н. Остапчук. ФТТ 55, 95 (2013).

[10] S.M. Ohr. J. Appl. Phys. 43, 1361 (1973).

[11] S.M. Ohr. Phys. Status Solidi B 58, 613 (1973).

[12] H.A. Elliott. Proc. Cambridge Phil. Soc. 44, 522 (1948); 45, 621 (1949).

[13] F. Kroupa. Czech. J. Phys. B 10, 284 (1960).

[14] П.Н. Остапчук, О.Г. Троценко. ФТТ 58, 1749 (2016).

[15] M.H. Yoo. Phys. Status Solidi B 61, 411 (1974).

[16] L. Fast, J.M. Wills, B. Johansson, O. Eriksson. Phys. Rev. B 51, 17431 (1995).

[17] C.H. Woo. J. Nucl. Mater. 276, 90 (2000).

[18] С.А. Кукушкин, А.В. Осипов, Р.С. Телятник. ФТТ 58, 941 (2016).

[19] V.I. Dubinko, A.S. Abyzov, A.A. Turkin. J. Nucl. Mater. 336, 11 (2005).

[20] T. Jourdan. J. Nucl. Mater. 467, 286 (2015).

[21] В.Ф. Зеленский, И.М. Неклюдов, Т.П. Черняева. Радиационные дефекты и распухание металлов. Наук. думка, Киев. (1988). $296 \mathrm{c}$.

[22] P.T. Heald, M.V. Speight. Phil. Mag. 29, 1075 (1974).

[23] L.K. Mansur. Phil. Mag. A 39, 497 (1979).

[24] V.I. Dubinko, S.A. Kotrechko, V.F. Klepikov. Rad. Eff. Defects Solids. 164, 647 (2009). 